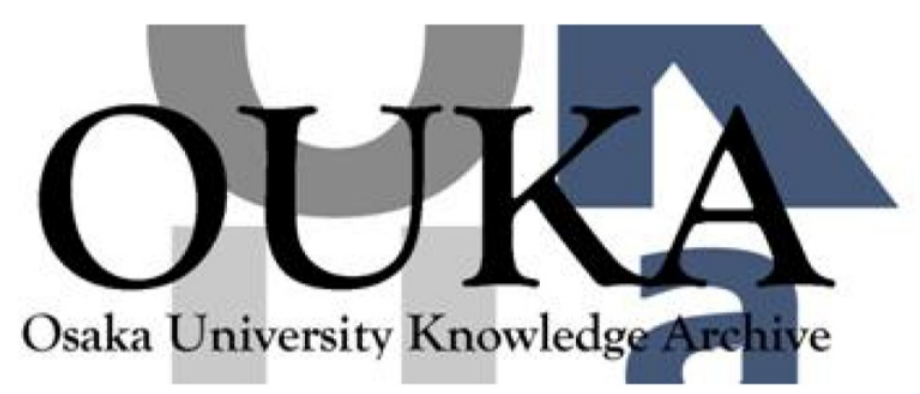

\begin{tabular}{|c|c|}
\hline Title & $\begin{array}{l}\text { Wave structure analysis of guided waves in a } \\
\text { bar with an arbitrary cross-section }\end{array}$ \\
\hline Author (s) & $\begin{array}{l}\text { Hayashi, Takahiro; Tamayama, Chiga; Murase, } \\
\text { Morimasa }\end{array}$ \\
\hline Citation & Ultrasonics. $44(1)$ p. 17-p. 24 \\
\hline Issue Date & $2006-01$ \\
\hline oaire:version & AM \\
\hline URL & https://hdl. handle. net/11094/84496 \\
\hline rights & $\begin{array}{l}\text { () } 2005 \text { Elsevier B.V. This manuscript version is } \\
\text { made avai lable under the Creative Commons } \\
\text { Attribution-NonCommercial-NoDerivatives } 4.0 \\
\text { International License. }\end{array}$ \\
\hline Note & \\
\hline
\end{tabular}

Osaka University Knowledge Archive : OUKA

https://ir. Library. osaka-u. ac. jp/

0saka University 


\section{Wave structure analysis of guided waves in a bar with an arbitrary cross-section}

Takahiro Hayashi, Chiga Tamayama and Morimasa Murase

Corresponding author: T. Hayashi

Faculty of Engineering, Nagoya Institute of Technology

Gokiso Showa Nagoya, 466-8555 Japan

Tel: +81-52-735-5338, Fax: +81-52-735-5338

E-mail: hayashi@nitech.ac.jp 
Hayashi Ultrasonics 2.

\begin{abstract}
Both dispersion curves and wave structures, which are displacement distributions on a bar cross-section, are essential for guided wave NDEs. Theoretical dispersion curves and wave structures for a bar with an arbitrary cross-section are derived in this paper using a special modeling technique called the Semi-Analytical Finite Element Method (SAFEM). The guidelines for guided wave NDEs of bar-like structures are also shown based on wave structure and modal analysis. First, the relationship between the dispersion curves and their corresponding wave structures were obtained for a square rod. Modes with longitudinal vibration have higher group velocities and torsional modes have constant phase and group velocities. Next, the relationship between the dispersion curves and wave structures for a rail are detailed. The rail is used to represent a bar with a complex cross-section. Similar to the square rod results, the rail's longitudinal modes have higher group velocities. However, the rail contains modes with local vibration. Finally, single mode detection and excitation techniques are introduced. A single mode can be obtained by detecting and exciting with a weighted function that corresponds to a specific mode's wave structure.
\end{abstract}

PACS numbers: 43.20. Jr, 43.20. Mv.

Keywords: guided wave, dispersion curve, wave structure, semi-analytical finite element method 
Hayashi Ultrasonics 3.

\section{Introduction}

Guided waves in bars or plate-like structures resonate between the boundaries of their cross-sections, and propagate in the longitudinal direction. Because the energy of guided waves does not spread like bulk waves, they have the potential for use in longrange propagation and rapid long-range inspection applications. Although guided wave inspection could possibly become an epoch-making technique, current theoretical difficulties, such as multi-mode and dispersion characteristics, prevent the technique from being incorporated into a variety of practical applications.

Since Rayleigh and Lamb presented their pioneering theoretical studies on acoustic waves in a bounded object more than one hundred years ago, researchers have continued to study guided waves in a plate, such as Lamb and shear horizontal plate waves. The use of guided waves in non-destructive evaluation (NDE) became an attractive and widely used technique after Viktrov [1] and Rose's [2] textbooks were published. These textbooks were based on Mindlin [3] and Onoe's [4] research in the 1970's on dispersion curves and wave structures, which are terms used to characterize the frequency dependence of guided wave velocities and displacement (or stress) distributions in a bar cross-section, respectively. In 1958, Gazis [5] first presented a theoretical study on the dispersion curves for a pipe. Following this, many researchers such as Cawley, Lowe, Alleyne and Rose et.al. [6-9] continued to theoretically and experimentally study dispersion curves and wave structures in more detail for the purpose of guided wave pipe inspection. As a result, pipe inspection tools that utilize guided waves have recently been developed [10-12]. 
Thus, in order to establish an NDE technique that utilizes guided waves, detailed theoretical studies on dispersion curves and wave structures are needed. However, very few theoretical studies on guided waves in a bar with an arbitrary cross-section, like railroad rails, have been established specifically for guided wave NDE. This is because a modeling technique is required to obtain dispersion curves for a bar with an arbitrary cross-section, unlike for simple structures like plates and pipes that can be obtained analytically. Before detailed theoretical studies were conducted, Rose et al. [13] experimentally presented the potential for using guided waves to inspect railroad rails. In this experiment, an impact hammer produced guided waves in a rail that could be detected nearly 2000 feet away from the source. After considering the noise level and attenuation rate, they concluded that guided wave signals could be detected up to 7000 feet away from the source. Wilcox et al. [14] developed a guided wave inspection system that consisted of a dry-coupling transducer array, a device for pressurizing dry-coupling transducers uniformly along the rail surface, signal excitation and detection devices, and signal processing and interface software. They presented a defect characterization technique based on guided wave structural analysis. Using this technique, the reflection echoes from different types of defects produce totally different wave structures. More detailed dispersion curve and wave structure analyses are needed to accurately characterize defects using the guided wave NDE.

The phase and group velocity theoretical dispersion curves for a square bar and a rail were obtained using the semi-analytical finite element method (SAFEM) [15]. The SAFEM involves dividing the cross-section of a bar-like structure into small elements. The displacement distribution is then represented by nodal displacements, an 
interpolation function on the cross-section, and an orthogonal function $\exp \left(i \xi_{z}\right)$ in the propagation direction $(z)$. The governing equations obtained from this method become an eigensystem, and the solution of the eigensystem reveals the eigenvalues $\xi$ that correspond to the wave number of the resonant modes at a certain frequency. The dispersion curves obtained by the SAFEM were verified by comparing them with experimental dispersion curves obtained by a two-dimensional fast Fourier transform. The results revealed that numerous modes exist in bar-like structures with complex crosssections and that the dominant modes are strongly dependent upon the transducers' input and receiving setup.

The previous paper [15] described the derivation of the theoretical dispersion curves in detail, but the theoretical wave structure analyses were not included. Considering that the dominant modes vary with measurement conditions, it follows that the interaction between guided wave propagation and defects should be strongly influenced by the wave structures of the guided wave modes. This study, therefore, investigates the relationship between wave structures and phase and group velocity dispersion curves, and details the guidelines for NDE using guided waves based on single mode detection and excitation.

\section{Brief description on Semi-analytical FEM}

Because the derivation of the SAFEM was detailed in [15], only the equations used in this paper will be briefly explained. When the cross-section of a bar-like material is divided into small elements, and the displacement field in the longitudinal (z) direction 
is expressed by the orthogonal function $\exp (i \xi z)$, the virtual work principle yields the following governing equations, similar to an ordinary FEM,

$\overline{\mathbf{f}}=\left(\mathbf{K}_{1}+i \xi \mathbf{K}_{2}+\xi^{2} \mathbf{K}_{3}\right) \overline{\mathbf{U}}-\omega^{2} \mathbf{M} \overline{\mathbf{U}}$,

In this equation, $\overline{\mathbf{f}}$ and $\overline{\mathbf{U}}$ are Fourier transform expressions with respect to the $z$ direction of a nodal force vector $\mathbf{f}$ and a displacement vector $\mathbf{U}$, respectively. $\mathbf{K}_{1}, \mathbf{K}_{2}$, $\mathbf{K}_{3}$ and $\mathbf{M}$ are regular matrices obtained from the material properties and geometry, $\xi$ is the wave number and $\omega$ is the angular frequency. Suppose that $M$ represents three times the number of nodes. The number of elements in the vectors in Eq. (1) is $M$, and the matrices in Eq. (1) are MxM. Eq. (1) can then be rewritten as the following first order eigensystem with respect to the wave number $\xi$,

$$
(\mathbf{A}-\xi \mathbf{B}) \mathbf{Q}=\mathbf{p}
$$

$\mathbf{A}=\left[\begin{array}{cc}0 & \mathbf{K}_{1}-\omega^{2} \mathbf{M} \\ \mathbf{K}_{1}-\omega^{2} \mathbf{M} & i \mathbf{K}_{2}\end{array}\right]$

$\mathbf{B}=\left[\begin{array}{cc}\mathbf{K}_{1}-\omega^{2} \mathbf{M} & 0 \\ 0 & -\mathbf{K}_{3}\end{array}\right]$

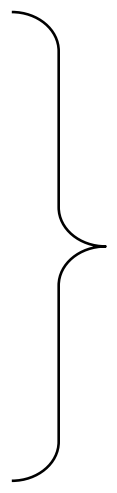

$\mathbf{Q}=\left[\begin{array}{c}\overline{\mathbf{U}} \\ \xi \overline{\mathbf{U}}\end{array}\right], \quad \mathbf{p}=\left[\begin{array}{l}0 \\ \overline{\mathbf{f}}\end{array}\right]$.

Assuming $\mathbf{p}=0$, the solution to the eigenvalue problem gives $2 M$ eigenvalues $\xi_{m}$ and $2 M$ right and left eigenvectors, $\boldsymbol{\Phi}^{R}{ }_{m}(2 M \times 1$ vector $)$ and $\boldsymbol{\Phi}^{L}{ }_{m}(1 \times 2 M$ vector $)$, where $m=1,2 \ldots 2 M$. These eigenvalues contain $M$ forward waves and $M$ backward waves.

This section addresses the characteristics of the right and left eigenvectors $\boldsymbol{\Phi}^{R}{ }_{m}$ and $\boldsymbol{\Phi}^{L}{ }_{m} . \boldsymbol{\Phi}^{R}{ }_{m}$ is a right eigenvector obtained from $\mathbf{A}-\xi \mathbf{B}$, and $\boldsymbol{\Phi}^{L}{ }_{m}$ is a conjugate 
transpose of $\boldsymbol{\Phi}^{L}{ }_{m}^{T}$ that is the right eigenvector of $\mathbf{A}^{T}-\xi^{*} \mathbf{B}^{T}$. Here $*$ and $T$ denote conjugate and conjugate transpose of a vector or matrix, respectively. Using Eq. (16) from the previous paper [15] and the symmetry of the elastic constant matrix $\mathbf{c}, \mathbf{K}_{1}, \mathbf{K}_{3}$ and $\mathbf{M}$ are symmetric matrices and $\mathbf{K}_{2}$ is an anti-symmetric matrix. Therefore, $\mathbf{A}$ and $\mathbf{B}$ are Hermitian matrices, that is,

$\mathbf{A}-\boldsymbol{\xi} \mathbf{B}=\mathbf{A}^{T}-\xi \mathbf{B}^{T}$

Using this property, the following relationship can be obtained between the right and left eigenvectors for propagating modes with real wave number $\xi$,

$\boldsymbol{\Phi}^{R}{ }_{m}=\boldsymbol{\Phi}_{m}^{L}{ }^{T}$

Using the form of $\mathbf{Q}$ in Eq. (3), the right eigenvector $\boldsymbol{\Phi}^{R}{ }_{m}$ can be expressed as

$\boldsymbol{\Phi}_{m}^{R}=\left[\begin{array}{c}\boldsymbol{\varphi}^{R}{ }_{m} \\ \xi \boldsymbol{\varphi}^{R}{ }_{m}\end{array}\right]$,

where $\varphi^{R}{ }_{m}(M \times 1$ vector $)$ is the upper part of $\boldsymbol{\Phi}^{R}{ }_{m}$. Using the orthogonality of eigenvector $\boldsymbol{\Phi}^{R}{ }_{m}$ :

$\boldsymbol{\Phi}^{R}{ }_{m}^{T} \boldsymbol{\Phi}_{n}^{R}=\left\{\begin{array}{cc}\boldsymbol{\Phi}^{R}{ }_{m}{ }^{T} \boldsymbol{\Phi}^{R}{ }_{m} & m=n \\ 0 & m \neq n\end{array}\right.$,

then, considering Eq. (6),

$\boldsymbol{\varphi}^{R}{ }_{m}^{T} \varphi^{R}{ }_{n}=\left\{\begin{array}{cc}\varphi^{R}{ }_{m}^{T} \varphi^{R}{ }_{m} & m=n \\ 0 & m \neq n\end{array}\right.$.

is satisfied for $\varphi^{R}{ }_{m}$. Similarly,

$\boldsymbol{\varphi}^{L}{ }_{m}=\varphi^{R}{ }_{m}^{T}$,

so the left eigenvectors satisfy the following equations: 


$$
\begin{gathered}
\boldsymbol{\Phi}^{L}{ }_{m}=\left[\begin{array}{ll}
\boldsymbol{\varphi}^{L}{ }_{m} & \xi \boldsymbol{\varphi}^{L}{ }_{m}
\end{array}\right] \\
\boldsymbol{\Phi}^{L}{ }_{m} \boldsymbol{\Phi}^{L}{ }_{n}{ }^{T}=\left\{\begin{array}{cc}
\boldsymbol{\Phi}^{L}{ }_{m} \boldsymbol{\Phi}^{L}{ }_{m}{ }^{T} & m=n \\
0 & m \neq n
\end{array}\right. \\
\boldsymbol{\varphi}^{L}{ }_{m} \boldsymbol{\varphi}^{L}{ }_{n}{ }^{T}=\left\{\begin{array}{cc}
\boldsymbol{\varphi}^{L}{ }_{m} \boldsymbol{\varphi}^{L}{ }_{m}{ }^{T} & m=n \\
0 & m \neq n
\end{array}\right.
\end{gathered}
$$

The relationships between the above eigenvectors and the nodal displacement vector $\mathbf{U}$ can be applied to a point source at $\mathrm{z}=\mathrm{ZS}_{\mathrm{S}}$ :

$$
\begin{gathered}
\mathbf{U}=\sum_{m=1}^{M} \alpha_{m} \boldsymbol{\varphi}^{R}{ }_{m} \exp \left\{i \xi_{m}\left(z-z_{S}\right)\right\} \\
\alpha_{m}=-\frac{\xi^{L}{ }_{m} \mathbf{f}}{B_{m}}, B_{m}=\boldsymbol{\Phi}_{m}^{L} \mathbf{B} \boldsymbol{\Phi}^{R}{ }_{m} .
\end{gathered}
$$

These equations indicate that the displacement vector is the summation of the wave structure vector at $z=z_{\mathrm{S}} \varphi^{R}{ }_{m}$ for resonant modes $m=1-M$ with weighted constants $\alpha_{m}$. This exponential function describes the phase shift from $\mathrm{z}=\mathrm{z}_{\mathrm{S}}$ for the $m$ th mode, where $m=1-M$. In this equation, $M$ represents the modes propagating forward, and $\alpha_{m}$ is the amplitude for $m$ th mode determined by the external force vector $\mathbf{f}$.

\section{Wave structures in a square rod}

Fig. 1 shows the phase velocity and group velocity dispersion curves for a square steel rod with longitudinal wave velocity $c_{L}=5.85 \mathrm{~mm} / \mu \mathrm{s}$ and transverse wave velocity $c_{T}=3.23 \mathrm{~mm} / \mu \mathrm{s}$. The phase velocity dispersion curves were obtained by calculating the wave numbers $\xi_{m}$ as eigenvalues of Eq. (2) for all frequency steps, and then converting them into phase velocities using $c=\xi_{m} / \omega$. The group velocity dispersion curves were 
obtained using the procedure detailed in [15]. The horizontal axes for both figures represent the non-dimensional frequency $\Omega\left(=f h / c_{T}\right)$, and the vertical axes are the nondimensional phase velocity $c / c_{T}$ and group velocity $c_{g} / c_{T}$, respectively, where $f$ is frequency and $h$ is the length of a side of the square cross-section. The wave structure vector $\varphi^{R}{ }_{m}$, a complex value, was obtained for all of the phase velocity dispersion curve data points. Then, the wave structure on a given cross-section can be animated by calculating $\varphi^{R}{ }_{m} \exp (-i \omega t)$ and varying time $t$. Fig. 2 displays the wave structures for eight representative points in the Fig. 1 dispersion curves. The wave structures at maximum displacement and the original cross-section (zero displacement) are both shown to provide a reference. Fig. 2(a)-(d) displays the Fig. 1(a)-(d) wave structures when the group velocity is $c_{g} / c_{T}>1$. Fig. 2(e) and (f) represent wave structures on a curve similar to a Lamb wave's A0 mode. The curve starts from zero velocity, monotonically increases as $f$ increases, and then approaches the Rayleigh wave velocity $c_{R}\left(\approx 0.93 c_{T}\right)$ as $f \rightarrow \infty$. Fig. $2(\mathrm{~g})$ and $(\mathrm{h})$ represent non-dispersive modes with almost constant wave velocities of $c, c_{g} \approx 0.92 c_{T}$. Longitudinal vibration dominates the faster modes with higher group velocities (a)-(d). The entire cross-section of (a) vibrates uniformly, while (b) contains one nodal point at the center of the cross-section. The cross-sections of (c) and (d) contain four and nine nodal points, respectively. Both (e) and (f) are flexural modes that vibrate the entire cross-section in the diagonal direction. The other flexural modes with exactly the same phase and group velocities but with different wave structures exist at the points on the dispersion curves contained in (e) and (f). These wave structures, however, are symmetric with respect to the cross-section's centerline. Considering the symmetry of a square rod's cross-section, it is expected that these 
symmetrical modes have the same phase and group velocities. Both (g) and (h) are torsional modes. From these two figures, it is apparent that the form of the square crosssection changes with higher frequency due to the larger inertia of the corners. Unlike the torsional mode for a pipe, the torsional mode for a square bar does not exhibit perfectly constant phase and group velocities [15,17-19].

The wave structures at the other locations are complex and exhibit mixed modes with flexural, torsional and longitudinal vibrations.

\section{Dispersion curves and wave structures for a rail}

The theoretical dispersion curves for the rail shown in Fig. 3 were derived in the previous paper [15] using SAFEM and verified through a comparison with experimental dispersion curves. In this case, the wave structures are obtained for the same rail geometry. Fig. 4 displays the theoretical dispersion curves. Compared to the square bar in Fig. 1, the dispersion curves in Fig. 4 contain a large number of modes in the given frequency range. Five representative wave structures from the $50 \mathrm{kHz}$ frequency were selected from the dispersion curves in Fig. 4 and are shown in Fig. 5 (a)-(e).

Points (a) and (b) in the Fig. 4 phase velocity dispersion curves resemble the A0 mode of a Lamb wave, but with slightly different velocities. Looking at the wave structures in Figs. 5 (a) and (b), these two points are flexural modes vibrating at different locations of the rail cross-section: part of the foot and the web, respectively. Both modes vibrate in a plate-like region with a uniform displacement distribution in the thickness direction. Because a similar flexural vibration is seen in the A0 mode of a Lamb wave, the dispersion curves of these two modes resemble the A0 mode's dispersion curve. The 
thickness differences in the plate-like regions can be attributed to the differences in phase velocity.

In Fig. 5(c), the railhead is moving in the diagonal direction due to flexural vibration in the web. However, there is no deformation in the head. Fig. 5(d) represents the mode involving compression and dilation to the right and left in the railhead. Mode (e) experiences much higher group velocity than transverse wave velocity $c_{T}$, and is dominated by longitudinal vibration, like in the square rod case. The entire rail crosssection vibrates in the longitudinal direction and boundary nodal lines exist between the railhead and the web and between the web and the foot.

A number of similarities and differences between the square rod and the rail were noted. For both cross-sectional shapes, the modes with longitudinal vibration have higher group velocity than transverse wave velocity. In addition, the flexural modes that vibrate in a plate-like region with uniform displacement in the thickness direction produce curves similar to the A0 mode of the Lamb wave. On the other hand, resonant modes that vibrate only one part of the cross-section were only evident in the rail structures, and the rail structures were not able to produce torsional modes with nearly constant velocities.

\section{Necessary receiving and loading conditions for single mode detection and excitation}

Because the wave structures are different for each mode, the interaction of each mode with defects in a bar-like structure should be different. Therefore, it is important to use a single mode detection and excitation technique to characterize defects using modal analysis. 
Multiplying both sides of Eq. (13) by $C \varphi^{R}{ }_{m}$ and utilizing the orthogonality of $\boldsymbol{\varphi}^{R}{ }_{m}$ described in Eq.(8), Eq.(13) becomes

$C \boldsymbol{\varphi}^{R}{ }_{m}^{T} \mathbf{U}=\alpha_{m} \varphi^{R}{ }_{m}{ }^{T} \boldsymbol{\varphi}^{R}{ }_{m} \exp \left\{i \xi_{m}\left(z-z_{S}\right)\right\}$

where $C$ is an arbitrary constant. The left side of Eq. (15) represents a series of waveforms measured at all nodes on cross-section $\mathbf{U}$ summed up with a weight function consisting of the right eigenvector for the $m$ th mode of $\varphi^{R}{ }_{m}$. The right side of Eq. (15) represents the extracted waveform of the $m$ th mode. So, if we can measure waveforms at all nods on the cross-section, the waveform obtained by summing up with a weight function corresponding to the wave structure of $m$ th mode gives the extracted signals for the $m$ th mode.

The loading conditions for single mode excitation are now considered. The nodal force vector is assumed to be defined as

$\mathbf{f}=C \boldsymbol{\varphi}^{R}{ }_{m}=C \boldsymbol{\varphi}^{L}{ }_{m}{ }^{T}$,

where $C$ is an arbitrary constant. Using the orthogonality of $\varphi^{L}{ }_{m}$ in Eq. (12), the amplitude of the $n$th mode $\alpha_{n}$ in Eq. (14) is

$\alpha_{n}=\left\{\begin{array}{cc}-C \frac{\xi \varphi^{L}{ }_{m} \varphi^{L}{ }_{m}{ }^{T}}{B_{m}} & m=n \\ 0 & m \neq n\end{array}\right.$.

This equation implies that when a nodal force $\mathbf{f}$ is applied on the cross-section $z=z_{S}$ with the distribution corresponding to the wave structure of $m$ th mode $\varphi^{R}{ }_{m}$, the single $m$ th mode is excited and the other unwanted modes are zero. As an example, the necessary 
nodal forces for exciting the modes in Figs. 5(a) and (b) are shown in Figs. 6(a) and (b), respectively. The arrows vary with time. After $1 / 4$ of a period, the necessary nodal forces are zero, and after $1 / 2$ of a period, all of the arrows point in the opposite direction.

In the previous exercise, both receiving and loading at a certain cross-section $\mathrm{z}=$ zs were considered. However, in practice, ultrasonic transducers can only be installed on the surface of bar-like structures, as shown in Fig. 7. In the case of a rail, the railhead surfaces and the web are accessible areas. Also, since only a limited number of transducers can be installed on the cross-section of a bar-like structure, a perfect single mode detection and excitation procedure is impossible.

In light of these restrictions, a single mode detection and excitation experiment can be conducted by arranging transducers in the longitudinal direction, as shown in Fig. 7. Using Eq. (13) to calculate the displacement distribution of the $m$ th mode in the $\mathrm{z}$ -

direction as $\exp \left\{i \xi_{m}\left(z-z_{S}\right)\right\}$, a phase shift of $\xi_{m} z_{S}$ should be applied to the transducers at $\mathrm{z}=\mathrm{z}$ to synchronize the signals. This is the same principle used by angle beam transducers, corm type EMAT and PVDF sensors for Lamb waves.

\section{Conclusions}

This paper used a square rod and a rail to examine dispersion curves and wave structures in bars with arbitrary cross-sections. A semi-analytical finite element method was employed, and the resulting characteristics were examined. In both cases, modes with higher group velocities than transverse wave velocities were dominated by longitudinal vibration. On the other hand, modes with lower group velocities were 
dominated by torsional and/or flexural vibrations. The rail structure cross-section also experienced some modes with partial vibration.

A single mode detection and excitation technique was also investigated. Single mode extraction is conducted by adding a series of waveforms measured on the crosssection of a bar-like structure with a weighted function corresponding to the wave structure of the necessary mode. A single mode can be excited by applying forces corresponding to the wave structure of the target mode. Even more efficient and practical detection and excitation of a single mode can be realized by considering the displacement distribution in the longitudinal direction.

\section{Acknowledgements}

The authors with to thank Prof. K. Kawashima and Prof. J. L. Rose for their encouragement for this work. This research was supported by Japan Institute of Construction Engineering under contract No. 03005. 


\section{Figure Captions}

FIG. 1. Dispersion curves for a square steel bar.

(a) phase velocity (b) group velocity

FIG. 2. Wave structures for a square bar at eight representative points on dispersion curves FIG.1.

(a) $\Omega=0.1, c / c_{T}=1.599$, uniform longitudinal vibration

(b) $\Omega=1.5, c / c_{T}=1.720$, longitudinal vibration with one nodal point

(c) $\Omega=2.6, c / c_{T}=1.808$, longitudinal vibration with four nodal points

(d) $\Omega=3.9, c / c_{T}=1.848$, longitudinal vibration with nine nodal points

(e) $\Omega=0.5, c / c_{T}=0.804$, flexural vibration at the lower frequency

(f) $\Omega=2.0, c / c_{T}=0.927$, flexural vibration at the higher frequency

(g) $\Omega=0.5, c / c_{T}=0.922$, torsional vibration with the lower frequency

(h) $\Omega=2.0, c / c_{T}=0.933$, torsional vibration with the higher frequency

FIG. 3. Rail geometry and sub-divisions

FIG. 4. Dispersion curves for rail (a) phase velocity, (b) group velocity

FIG. 5. Wave structures for a rail at five representative points on the dispersion curves FIG.4.

(a) $50 \mathrm{kHz}, c=2.28 \mathrm{~mm} / \mu \mathrm{s}$, (b) $50 \mathrm{kHz}, c=2.55 \mathrm{~mm} / \mu \mathrm{s}$, (c) $50 \mathrm{kHz}, c=2.97 \mathrm{~mm} / \mu \mathrm{s}$, (d)

$50 \mathrm{kHz}, c=3.52 \mathrm{~mm} / \mu \mathrm{s}$, (e) $50 \mathrm{kHz}, c=5.43 \mathrm{~mm} / \mu \mathrm{s}$

FIG. 6. Necessary nodal forces for a single mode excitation.

(a) For the mode in FIG.5 (a), (b) For the mode in FIG.5 (b)

FIG. 7. One example of the sensor set-up 


\section{FIG.1}

Takahiro HAYASHI

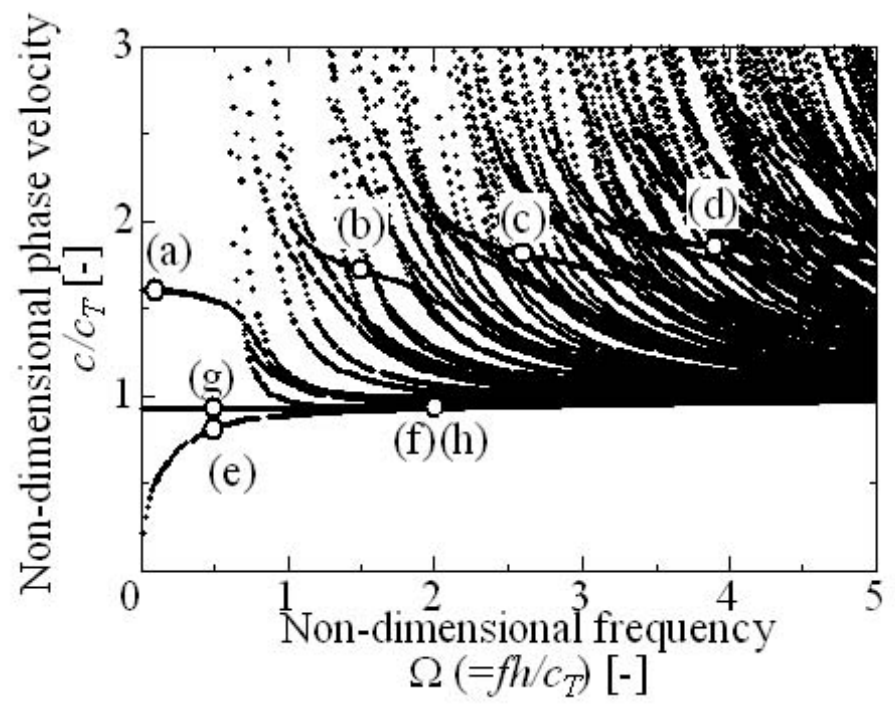

(a) phase velocity

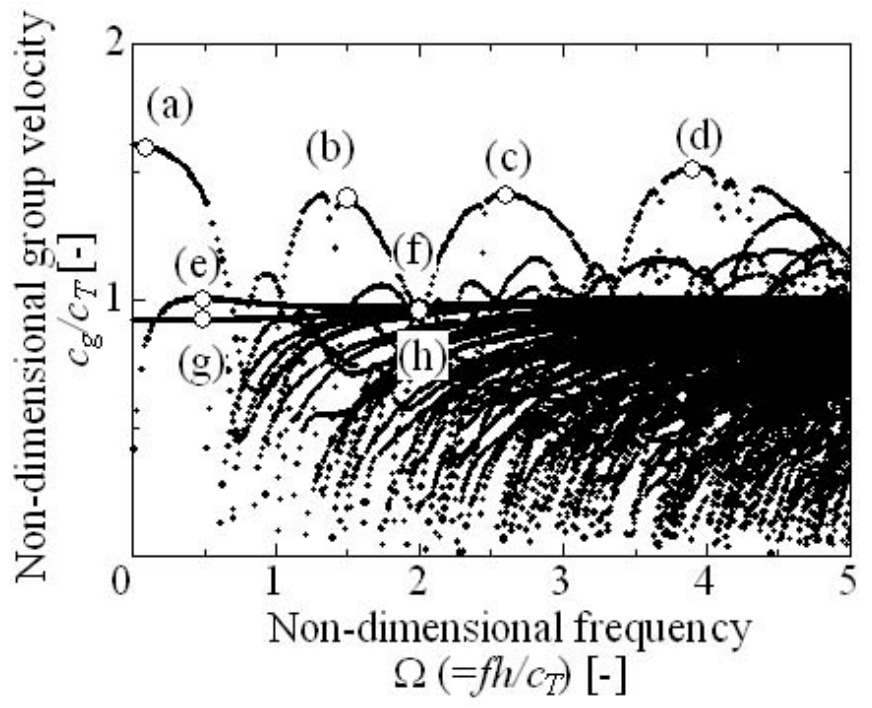

(b) group velocity 
Hayashi Ultrasonics, 19

\section{FIG.2}

Takahiro HAYASHI

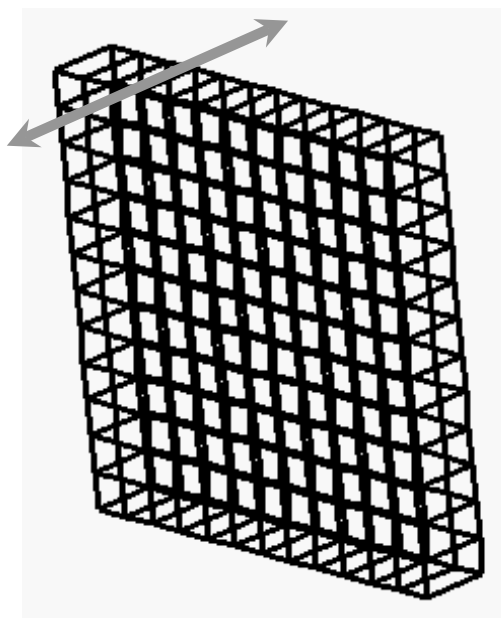

(a)

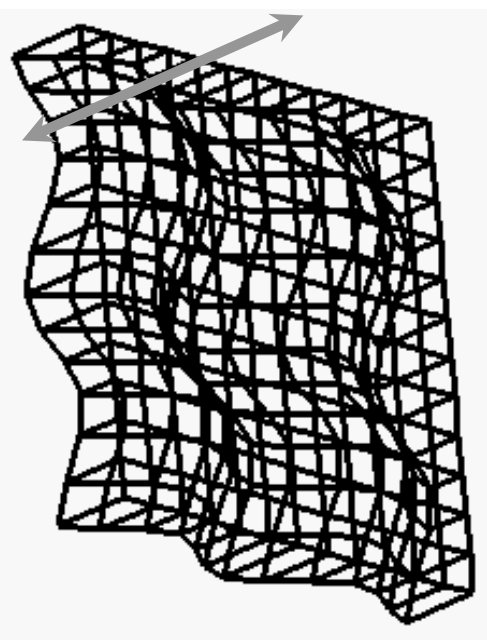

(c)

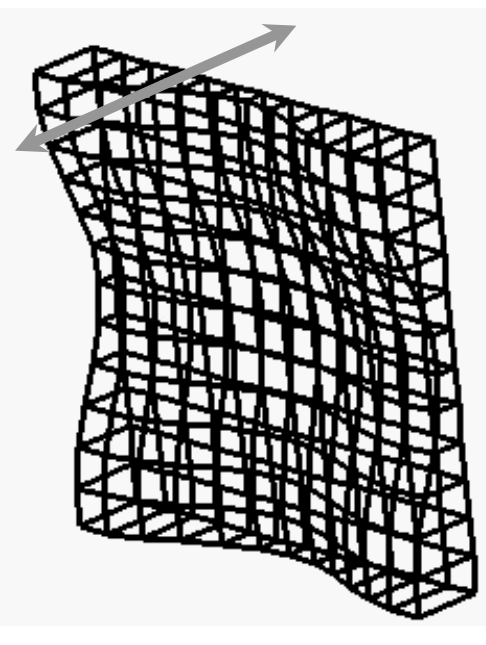

(b)

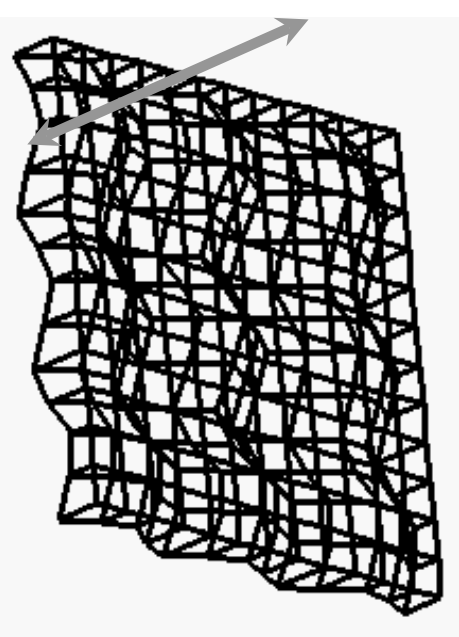

(d) 
FIG.2 contd.

Takahiro HAYASHI

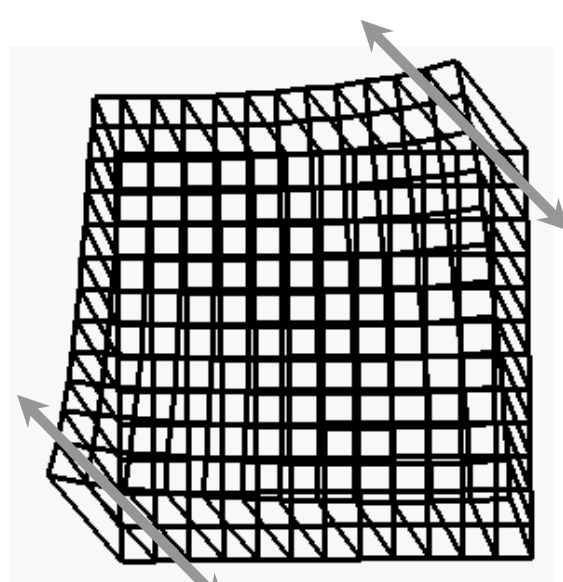

(e)

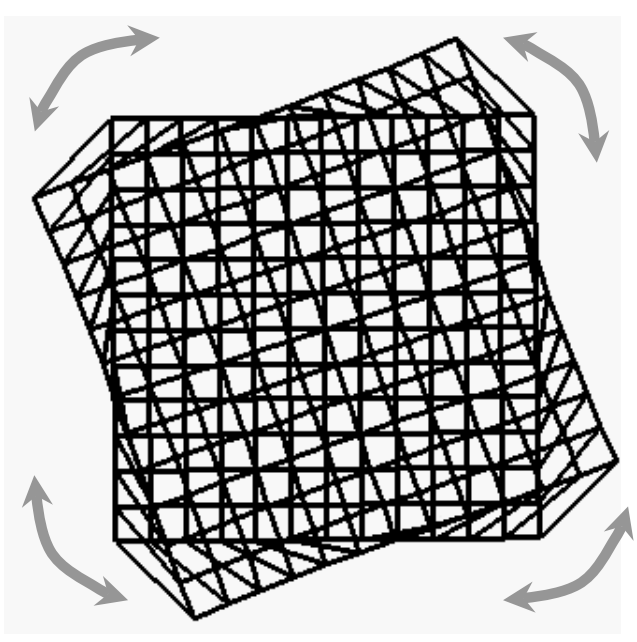

(g)

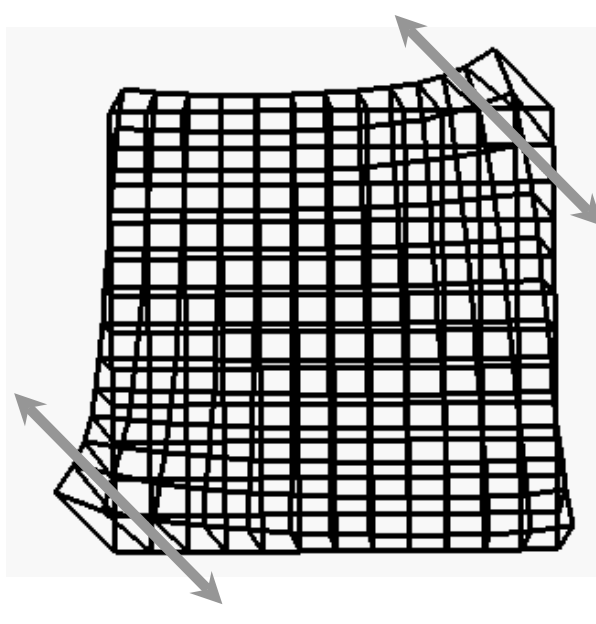

(f)

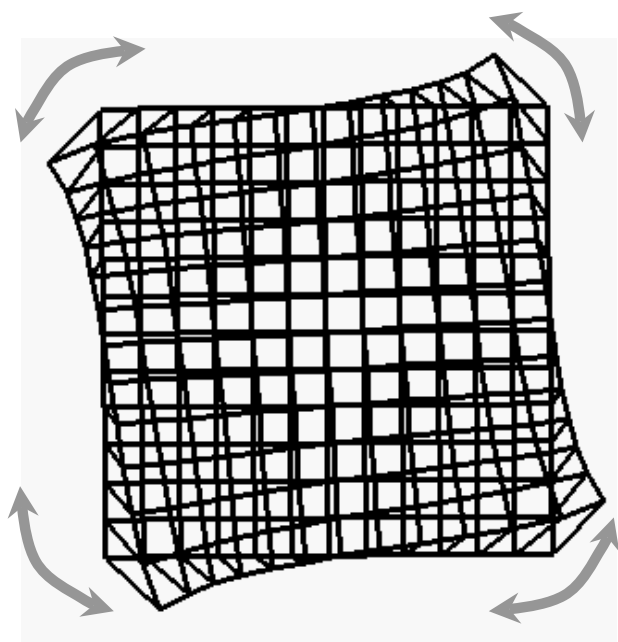

(h) 
Hayashi Ultrasonics, 21

\section{FIG.3}

Takahiro HAYASHI

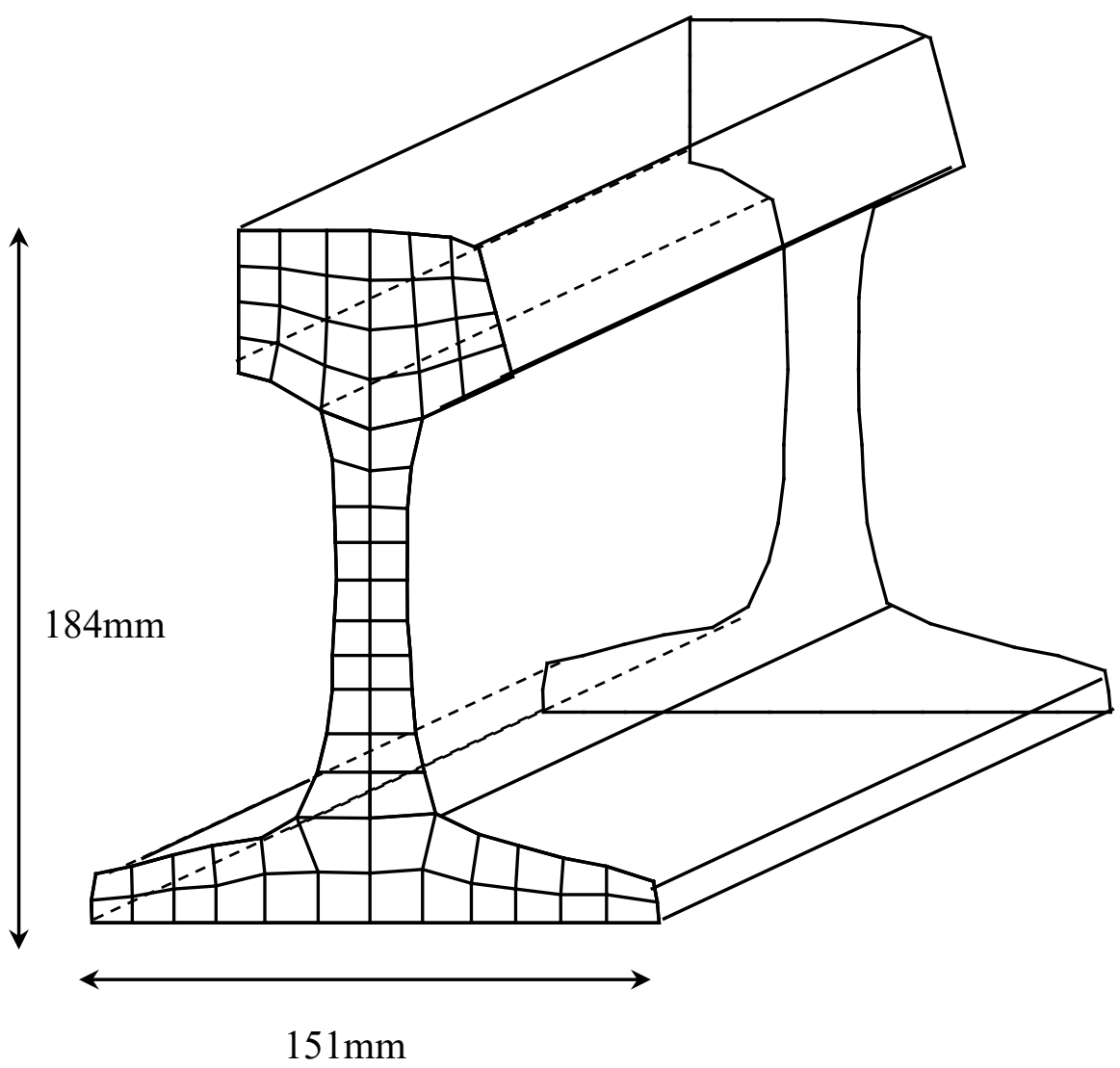




\section{FIG.4}

Takahiro HAYASHI

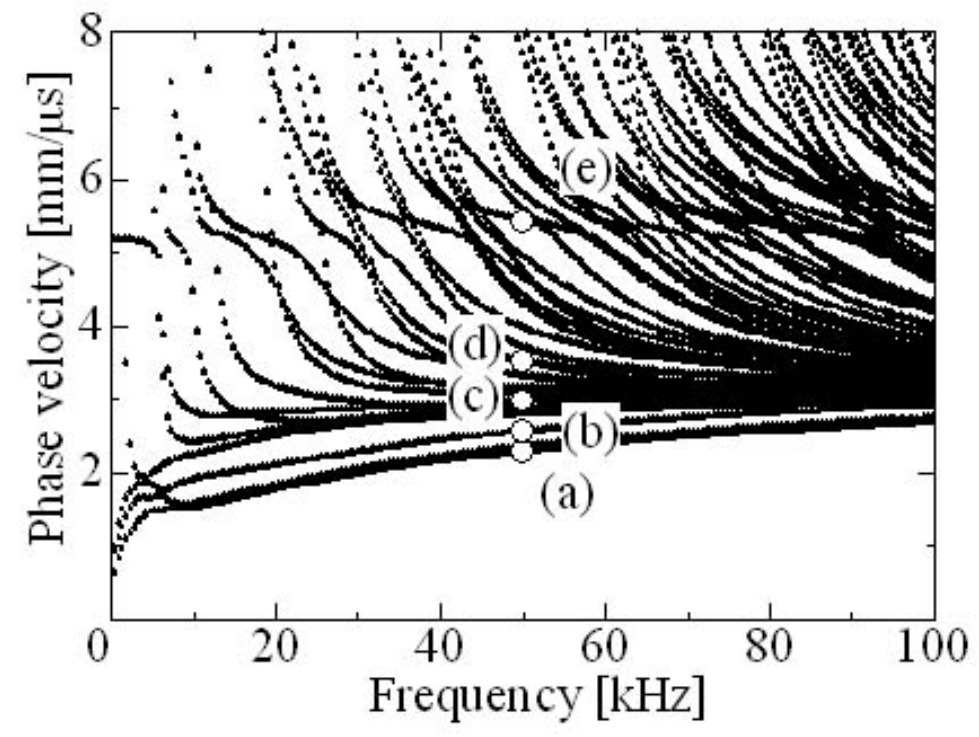

(a)

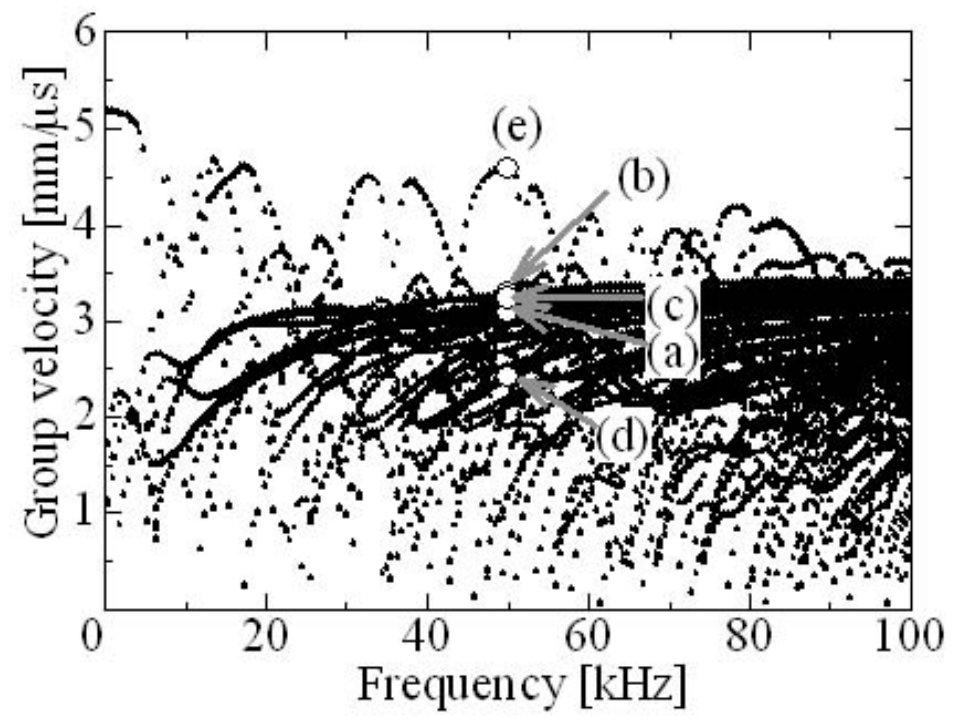

(b) 
Hayashi Ultrasonics, 23

\section{FIG.5}

Takahiro HAYASHI

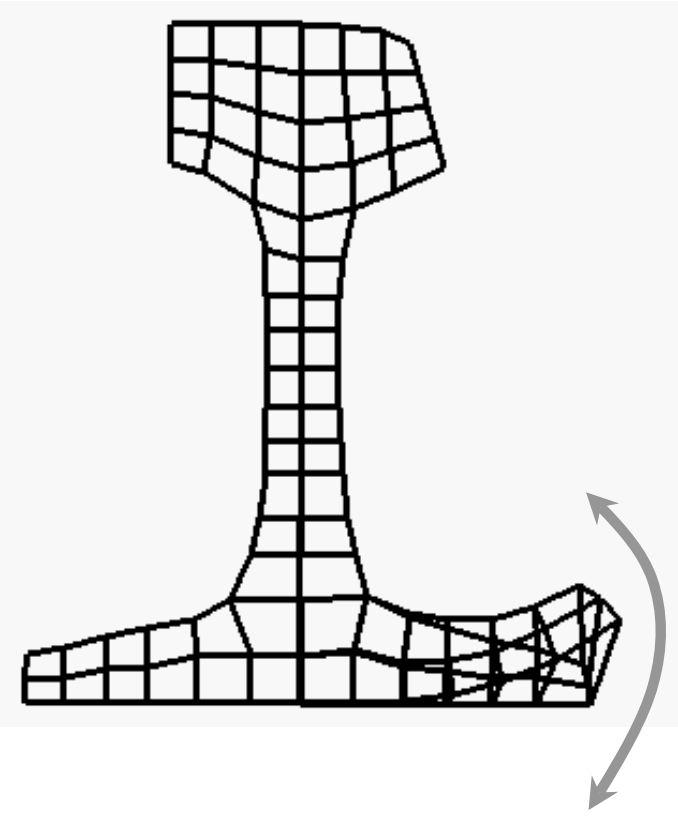

(a)

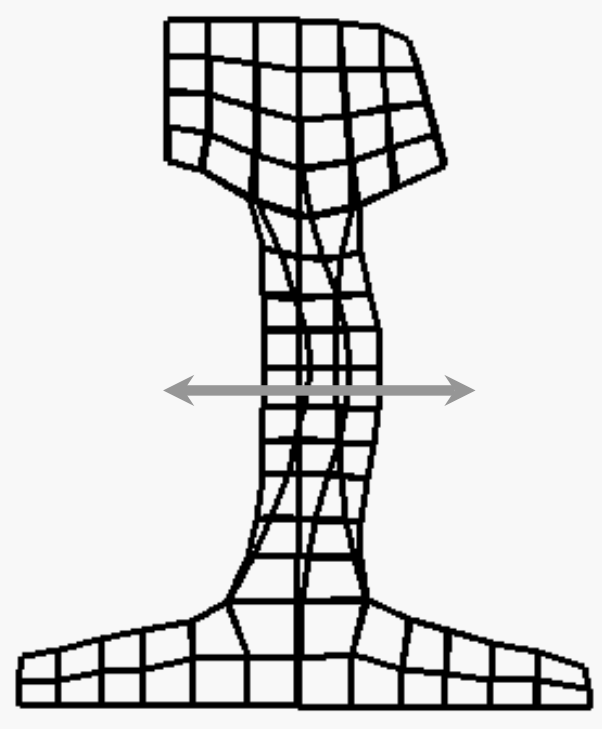

(b) 
Hayashi Ultrasonics, 24

FIG.5 contd.

Takahiro HAYASHI

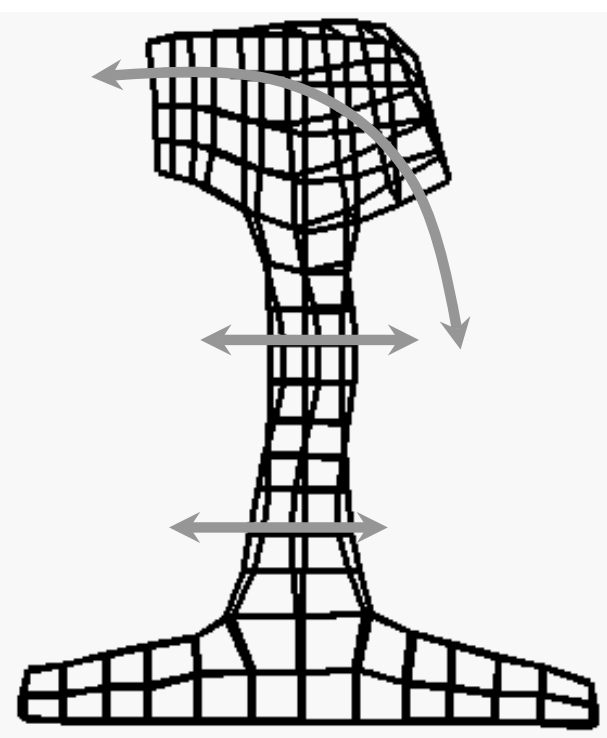

(c)

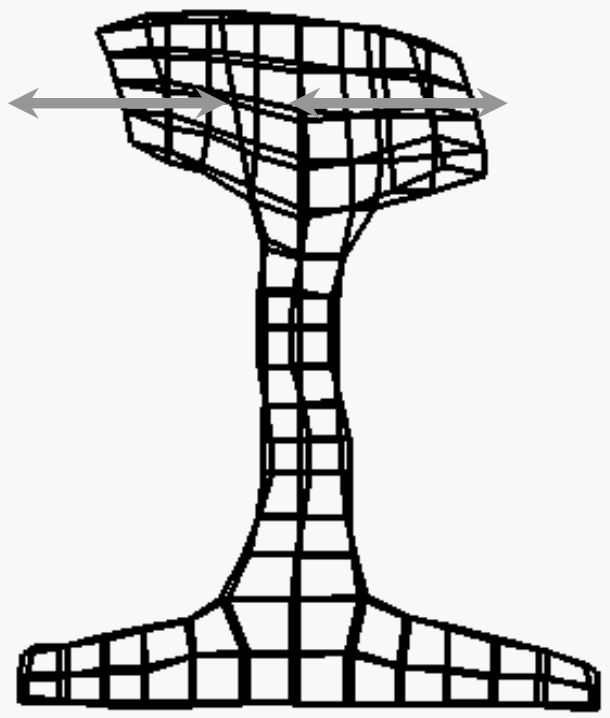

(d)

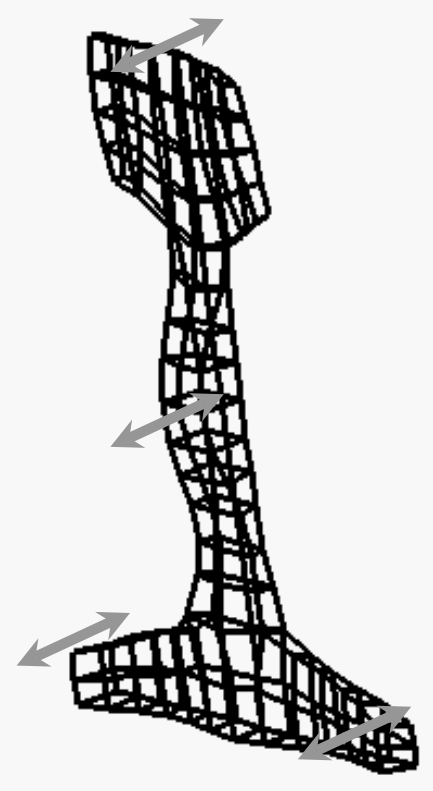

(e) 
Hayashi Ultrasonics, 25

\section{FIG.6}

Takahiro HAYASHI

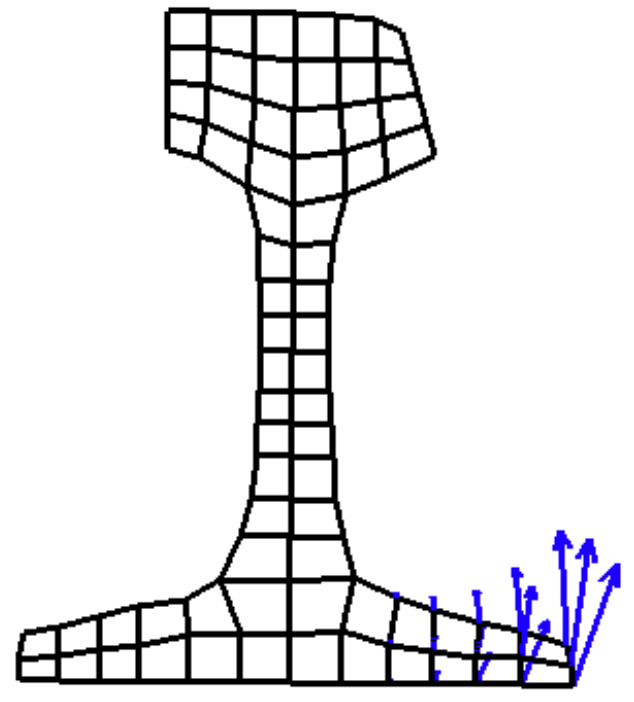

(a)

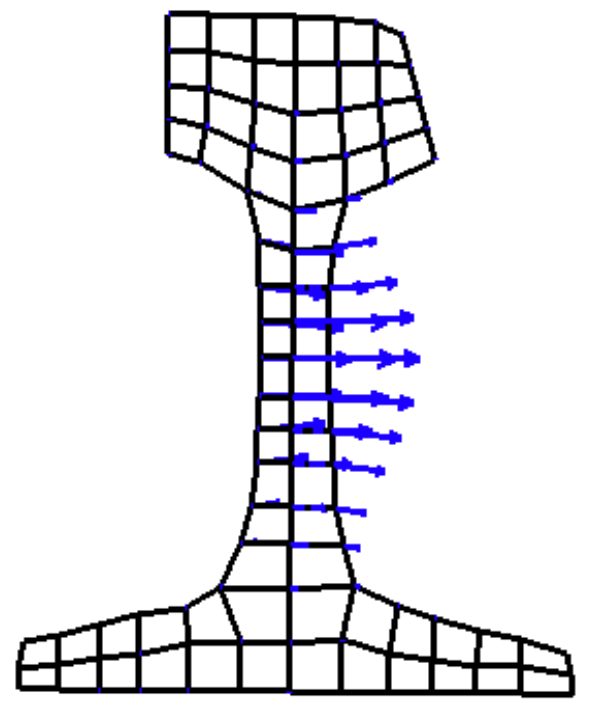

(b) 
Hayashi Ultrasonics, 26

\section{FIG.7}

Takahiro HAYASHI

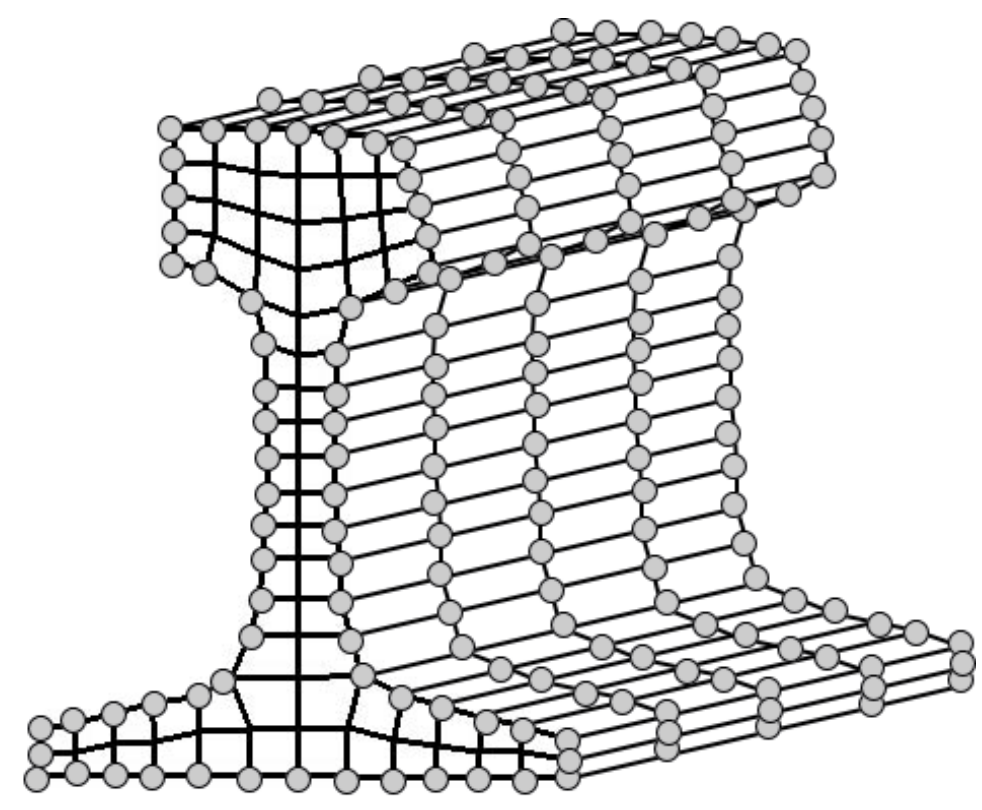




\section{References}

[1] R. D. Mindlin, Waves and vibrations in isotropic, elastic plates, Structural Mechanics, edited by J. N. Goodir and N. Hoff (Pergamon Press, Oxford 1959), 199-232

[2] M. Onoe, Contour vibrations of thin rectangular plates, J. Acoust. Soc. Am., 30, 12, (1962) 1159

[3] I. A. Victorov, Rayleigh and Lamb waves (Plenum Press, New York, 1967)

[4] J. L. Rose, Ultrasonic Waves in Solid Media (Cambridge University Press, 1999)

[5] D. C. Gazis, Three-Dimensional Investigation of the Propagation of Waves in Hollow Circular Cylinders. I. Analytical Foundation and II Numerical Results, J. Acoust. Soc. Am., 31 (5) (1959) 568-578

[6] P. Cawley, M. J. S. Lowe, D. N. Alleyne, B. Pavlakovic and P. Wilcox, Practical long range guided wave testing: Application to pipes and rails, Materials Evaluation, 61, (1) (2003) 66-74

[7] M. J. S. Lowe, D. N. Alleyne and P. Cawley, The mode conversion of a guided wave by a part-circumferential notch in a pipe, J. Appl. Mech, 65 (1998) 211-214

[8] D. N. Alleyne and P. Cawley Long range propagation of Lamb waves in chemical plant pipework, Materials Evaluation, 55 (1997) 504-508

[9] J. L Rose, A baseline and vision of ultrasonic guided wave inspection potential, Trans. ASME PVP, 124 (2002) 273-282

[10] D. N. Alleyne, B. Pavlakovic, M. J. S. Lowe and P. Cawley, Rapid long-range inspection of chemical plant pipework using guided waves, Insight, 43 (2001) 93-96 
[11] P. J. Mudge, Field application of the Teletest (R) Long-range ultrasonic testing technique, Insight, 43 (2001) 74-77

[12] H. Kwun, S. Y. Kim and G. M. Light, The magnetostrictive sensor technology for long range guided wave testing and monitoring of structures, Materials Evaluation, 61 (1) (2003) 80-84

[13] J. L. Rose, M. J. Avioli and W.-J. Song, Application and potential of guided wave rail inspection, Insight, 44, (6) (2002) 353-358

[14] P. Wilcox, M. Evans, B. Pavlakovic, D. N. Alleyne, K. Vine, P. Cawley and M. J. S. Lowe, Guided wave testing of rail, Insight, 45 (2003) 413-420

[15] T. Hayashi, W.-J. Song and J. L. Rose, Guided wave dispersion curves for a bar with an arbitrary cross-section, a rod and rail example, Ultrasonics, 41 (2003) 175-183

[16] G. R. Liu, J. D. Achenbach, Strip element method for stress analysis of anisotropic linearly elestic solids, J. Appl. Mech. 62 (1995) 607

[17] N. J. Nigro, Steady-state wave propagation in infinite bars of noncircular cross section, J. Acoust. Soc. Am., 40, 6, 1501-1508 (1966)

[18] B. Aalami, Waves in prismatic guides of arbitrary cross section, J. Appl. Mech., 40, 1067-1072 (1973)

[19] H. Taweel, S. B. Dong and M. Kazic, Wave reflection from the free end of a cylinder with an arbitrary cross-section, Int. J. Solids Structures, 37, 1701-1726 (2000) 